\title{
Labyrinthe
}

$6 \mid 2000$

Numéro 6

\section{La fabrique « bertillonienne » de l'identité}

Entre violence physique et symbolique

\section{Pierre Piazza}

\section{(2) OpenEdition}

\section{Journals}

Édition électronique

URL : http://journals.openedition.org/labyrinthe/453

DOI : $10.4000 /$ labyrinthe.453

ISSN : 1950-6031

Éditeur

Hermann

\section{Édition imprimée}

Date de publication : 1 juin 2000

Pagination : 33-50

\section{Référence électronique}

Pierre Piazza, «La fabrique « bertillonienne » de l'identité », Labyrinthe [En ligne], 6 | 2000, mis en ligne le 23 mars 2005, consulté le 30 avril 2019. URL : http://journals.openedition.org/labyrinthe/453 ; DOI : $10.4000 /$ labyrinthe.453

Ce document a été généré automatiquement le 30 avril 2019.

Propriété intellectuelle 


\section{La fabrique « bertillonienne » de l'identité}

Entre violence physique et symbolique

Pierre Piazza

Labyrinthe poursuit ici, sur le thème de « La fabrique de l'identité », la réflexion amorcée dans notre précédente livraison par Vincent Denis. Celui-ci, en étudiant le développement croissant des « papiers d'identification » dans la France des Lumières, y discernait l'élaboration balbutiante de nouveaux codes de l'identité individuelle. Ici, c'est

à un véritable saut temporel que Pierre Piazza nous invite : après l'Ancien Régime finissant, voici la III République déjà sûre d'elle-même. Surtout, le

« bertillonnage » tiré au cordeau dont il est question ne partage guère de point commun avec le maquis de feuilles volantes défriché précédemment : la fabrique de l'identité a elle aussi connu sa Révolution industrielle.

En 1879, un jeune commis aux écritures de la Préfecture de police de Paris élabore une nouvelle méthode qui, reposant sur la mesure de certaines parties du corps (longueur de la tête, du médius, de l'auriculaire, de la coudée, du pied, etc.), permet d'identifier avec une certitude quasi absolue n'importe quel individu. L'anthropométrie d'Alphonse Bertillon ne suscite pas d'emblée l'enthousiasme du préfet Louis Andrieux qui ne voit en son subordonné qu'un cas « d'aliénation mentale ${ }^{1}$ ». Pourtant, devenu, quelques années plus tard, responsable du service de l'identification des détenus puis du service de l'identité judiciaire de la Préfecture de police de Paris où, Bertillon rationalise, pour les adjoindre à ses mensurations, toutes les pratiques policières relatives à la photographie et au relevé des marques corporelles. Il fut vite apprécié par la grande majorité de ses 
contemporains comme un savant d'exception, au même titre que Louis Pasteur, et ses inventions saluées comme une illustration du génie français.

2 Le succès obtenu par le système anthropométrique ou " bertillonnage » doit se comprendre au regard de la forte demande sociale qui, au début de la III République, incite les autorités à se prononcer en faveur de la déportation des délinquants récidivistes hors du territoire métropolitain ${ }^{2}$. La publication de statistiques criminelles de plus en plus pessimistes ainsi que l'importance accordée par la presse aux crimes de sang ${ }^{3}$ et aux discours savants sur la criminologie sont quelques-uns des facteurs qui exacerbent ce problème et encouragent nombre d'hommes politiques à envisager une accentuation de la répression à l'encontre des individus décrits comme « incorrigibles » car soupçonnés de commettre constamment les mêmes forfaits ${ }^{4}$.

Adoptée par une forte majorité au Parlement, la loi du 27 mai 1885 sur la relégation et l'interdiction de séjour consacre législativement le durcissement de l'action qu'entend mener le régime républicain dans ce domaine. Dès lors, les découvertes d'Alphonse Bertillon se révèlent incontournables puisqu'elles apparaissent comme seules capables de répondre sérieusement à l'impératif d'identification qui conditionne toute la réussite de la politique de prophylaxie sociale dans laquelle s'engage le gouvernement ${ }^{5}$. À partir du milieu des années 1880 , les pouvoirs publics s'ingénient donc à rendre au plus vite opérationnel le système anthropométrique à la Préfecture de police et, rapidement, celuici leur permet de découvrir de nombreuses dissimulations et substitutions d'identité commises par ceux que A. Bertillon qualifie lui-même de « sauvages de notre civilisation ${ }^{6}$ ».

4 Dans le résumé de son cours "Sécurité, territoire et population » rédigé pour l'Annuaire du Collège de France, Michel Foucault insistait sur la nécessité de répertorier et d'étudier l'ensemble des " procédures et moyens mis en œuvre pour assurer, dans une société donnée, le "gouvernement des hommes" " ». Cette préoccupation sera aussi la nôtre : nous nous demanderons dans quelle mesure le bertillonnage a été mobilisé par la police pour imposer à une partie de la population une certaine forme de pouvoir. Notre propos est de souligner toute l'importance que revêtent les dispositifs bureaucratiques qui s'articulent aux débats législatifs mis en forme par le droit. Il convient en effet de ne jamais oublier que les discours politiques et les règles juridiques qui les sanctionnent « prennent corps dans des ensembles techniques [...] qui à la fois les imposent et les maintiennent ${ }^{8}$ ». À cet égard, le bertillonnage constitue un objet d'étude particulièrement intéressant car à travers celui-ci peut être perçue une transformation sensible de la violence exercée par l'État. Technologie d'identification policière qui relève de ce processus du " grand renfermement " que Michel Foucault s'est attaché à analyser, il renvoie aussi à l'émergence d'un mode de domination plus symbolique inhérent au monde démocratique moderne qui, selon Gérard Noiriel, ne peut pas être complètement compris par le biais de la seule problématique foucaldienne de la disciplinarisation de la société 9 .

5 Les différentes étapes du système anthropométrique instauré à la Préfecture de police de Paris correspondent à une méticuleuse prise en charge du corps. Elles le font entrer « dans une machinerie de pouvoir qui le fouille, le désarticule et le recompose ${ }^{10} »$. Tout est, dans les moindres détails, calculé et mis en œuvre par la police pour le maîtriser et le domestiquer afin d'en extraire plus facilement une vérité cachée car c'est avant tout le « corps qui parle ${ }^{11} »$. 
Chaque matin, les personnes détenues au Dépôt ${ }^{12}$ sont dirigées vers la Préfecture de police " par le $\operatorname{collet}^{13}$ ». Rigoureusement déterminé, ce cérémonial immuable constitue la première étape d'une véritable entreprise policière d'atomisation des corps. Comme le précise A. Bertillon, la direction du Dépôt prend soin de bien « livrer dans des escouades différentes les individus arrêtés le même jour pour la même affaire. Les personnes réunies ainsi par le hasard ne se connaissent donc pas et n'ont pas le temps matériel de faire connaissance $[. . .]^{14} »$. Une fois parvenus dans les locaux de l'identification judiciaire situés sur l'île de la Cité, défense est faite aux individus de communiquer entre eux : de grandes inscriptions murales leur rappellent que toute conversation est strictement prohibée. Avant d'être amenés dans les pièces où leur sont appliqués les procédés anthropométriques, ils patientent dans une salle où s'exerce une "surveillance à la fois globale et individualisante ${ }^{15}$ " qui n'est pas sans rappeler le panoptique de Jeremy Bentham : cette salle est « garnie de bancs tout autour, mais sur ces bancs, chaque place est séparée par deux cloisons qui forment une stalle isolée comparable à une guérite [...] Un [policier] municipal assis sur une chaise à l'extrémité de la salle surveille ${ }^{16}$ »».

7 Si elles peuvent apparaitre comme de banales prescriptions organisationnelles visant à optimiser le rendement journalier de son activité, ces mesures drastiques grâce auxquelles la police encadre et régit les conduites individuelles n'en demeurent pas moins révélatrices d'une stratégie plus globale qui s'efforce de trouver des solutions nouvelles en vue de juguler un fléau surtout appréhendé comme inquiétant car organisé. En effet, c'est essentiellement parce qu'elle est réputée constituer une masse indifférenciée sans cesse plus nombreuse, dense et structurée que la population criminelle et délinquante est considérée comme menaçante pour l'ordre social établi ${ }^{17}$. Pour la police, la dissoudre en la ramenant à une simple juxtaposition d'individus lui permet de mieux la comprendre et la combattre. Séparer, trier les présumés malfaiteurs, ne serait-ce que d'un point de vue spatial dans ses services, et leur imposer le silence, l'autorisent ainsi à interdire l'émergence de quelconques formes d'entraide ou de solidarité susceptibles de rendre plus difficile, voire de compromettre la réalisation de ses projets en matière d'identification.

Isolés et étroitement surveillés, les individus soumis aux méthodes anthropométriques doivent aussi se déshabiller partiellement : ils se présentent devant les opérateurs « les pieds nus, le paletot enlevé, le col de chemise déboutonné et les manches retroussées ${ }^{18}$ ». Obéissant à des considérations d'ordre pratique (la nécessité de relever des mesures avec le maximum de précision), cette obligation qui leur est faite révèle également une volonté délibérée de les placer dans une situation inconfortable pour les rendre plus dociles ${ }^{19}$. Les propos tenus par Alphonse Bertillon et rapportés ultérieurement par sa propre nièce tendent à le confirmer : " Un malfaiteur que j'ai réussi à mettre pieds nus, racontait-il plus tard, n'a plus la même brutalité ni la même arrogance que s'il avait gardé ses chaussures. Car, étant profond psychologue, il avait remarqué qu'il suffit de mettre un homme en état d'infériorité vis-à-vis de lui-même pour rendre timide le plus décidé. En effet, dès que le prisonnier était nu-pieds, il n'offrait aucune résistance aux employés de la mensuration ${ }^{20} »$.

9 Lorsque surviennent enfin les deux principales phases du bertillonnage, c'est-à-dire les mensurations et la photographie, les fonctionnaires de la Préfecture de police observent une batterie d'instructions dont le seul but est d'obliger chaque individu à se conformer à toute une série de mouvements et de postures strictement définis. Placé contre un mur les bras en croix, assis sur un tabouret de quarante centimètres de hauteur, debout sur un 
seul pied... Lors de la mesure des différentes parties de ses membres, l'individu fait l'objet d'une multitude d'attouchements auxquels il doit impérativement se soumettre. La confection des clichés signalétiques face/profil, " opération presque exclusivement mécanique ${ }^{21}$ ", engendre une même emprise physique et tend à l'instituer en automate qui ne peut que subir la froideur fonctionnelle des contraintes qu'on lui impose ${ }^{22}$. La conception et l'usage des appareils tout comme la normalisation des procédures ne lui laissent presque aucune autonomie : « La chaise où l'on fait asseoir les détenus pour les photographier a été construite d'après des indications spéciales [...]. Le siège et le dossier, entièrement en bois, sans crin ni ressorts, sont recouverts d'une rude étoffe, de façon à écarter toute cause d'affaissement du modèle pendant la pause ${ }^{23}$ ». Au supplice de cette " étonnante machine célibataire qui tient à la fois de la chaise électrique et de la guillotine ${ }^{24}$ » s'ajoute la longueur du temps d'exposition ainsi que le maintien de la tête de l'individu par une réglette en fer ou par un aide-opérateur qui, toutes proportions gardées, apparait, au même titre que le bourreau, comme « l'agent d'une violence qui s'applique, pour la maîtriser, à la violence du crime ${ }^{25}$ ».

Fort de l'accueil favorable réservé à ses méthodes par de très nombreux journaux ${ }^{26}, \mathrm{~A}$. Bertillon préconise même l'emploi de la force physique si les circonstances l'exigent : «L'approbation que la presse a bien voulu lui donner implique donc comme conséquence l'approbation de la contrainte corporelle, si besoin est ${ }^{27} »$. Dans la grande majorité des cas pourtant, son usage par la police ne s'avère pas indispensable car les inculpés savent qu'un refus trop énergique de se plier au bertillonnage renforcerait la présomption de culpabilité pesant sur eux. Leur résistance s'exprime donc plus subtilement. Elle consiste davantage à exploiter les failles supposées d'une implacable logique policière dont ils ne peuvent comprendre tous les ressorts. Certains essayent, par exemple, de fausser volontairement la prise de la mesure en ne restant pas immobiles ou en se courbant. Mais, la rigueur caractérisant l'agencement du dispositif policier de maîtrise de leur corps ne leur offre que d'infimes chances d'affecter ainsi l'exactitude des données résultant des diverses opérations anthropométriques. De tels artifices sont d'ailleurs d'autant plus faciles à déceler qu'ils sont prévus et ne peuvent revêtir qu'une dimension individuelle ${ }^{28}$. Parce que sérieusement formés, comme le montre le contenu des règles édictées par $\mathrm{A}$. Bertillon qu'ils respectent à la lettre, les fonctionnaires parviennent régulièrement à les faire échouer : «Pour les deux ou trois mensurations qui peuvent être modifiées par suite de tricherie ou de mauvaise volonté de la part du sujet, un bon exercice sera d'exécuter soi-même les mouvements qui peuvent altérer les résultats, et de les constater sur ses collègues, de façon à être à même de les déjouer facilement s'ils venaient à se produire dans la pratique ${ }^{29}$ ». De la même façon, toute tentative d'échapper à la rigidité de la codification du protocole photographique de la Préfecture de police demeure difficile. Face au déploiement d'une technologie qui les dépossède de leur propre image, les grimaces, froncements de sourcils et modifications de leur regard devant l'objectif sont autant de défis lancés aux opérateurs qui, s'ils perturbent momentanément leur action, n'en compromettent que très marginalement l'efficacité.

11 Si le bertillonnage s'apparente certes à ces techniques qui " assujettissent les corps, dirigent les gestes, régissent les comportements ${ }^{30}$ » et qui, pour être correctement appliquées, nécessitent quelquefois le recours à la coercition, il permet aussi à la police d'envisager une rupture radicale avec des pratiques dénoncées par son créateur comme archaïques car inopérantes et trop violentes. Pour A. Bertillon, la violence inhérente à ses inventions est au contraire tolérable car mieux dosée et canalisée au sein d'une 
institution où rien n'est laissé aux effets du hasard pour que s'affirme la supériorité logique de la science. Avec le système anthropométrique, il entend promouvoir une police plus raisonnée et raisonnable, plus soucieuse du respect des libertés individuelles et s'orientant résolument sur la voie de la professionnalisation ${ }^{31}$. Une police transparente, sans abus de pouvoir, dont le tout nouveau régime républicain n'aura plus à dissimuler les agissements. Par la mobilisation de méthodes et de techniques rationnellement élaborées, il souhaite surtout exclure autant que possible l'usage de la violence purement physique du répertoire d'action des services d'identification de la Préfecture de police. Un tel dessein suppose l'établissement de rapports moins intimes entre des individus érigés en objets de savoir et des policiers-savants disposant seuls de connaissances et de savoirfaire, inaccessibles aux profanes, à l'aide desquels ils peuvent imposer leur pouvoir sans brusquerie.

12 Les moyens jusqu'alors employés par la police en vue de reconnaître les récidivistes se réduisaient le plus souvent à des astuces ou à des ruses $^{32}$. Ces leurres impliquaient une inéluctable proximité entre des agents de police, héritiers des méthodes brutales inaugurées sous l'ère Vidocq, et des individus dénoncés comme marginaux dont il convenait de découvrir l'identité. Ils étaient à la source des nombreux mauvais traitements (passages à tabac notamment) infligés par des policiers significativement désignés par l'argot populaire sous le nom de « $\operatorname{cognes}^{33}$ » et ils inspiraient fréquemment à leurs « victimes » l'idée de tromper, en leur inculquant même parfois les techniques de la transgression ${ }^{34}$. À ces stratagèmes aléatoires et souvent cruels, Alphonse Bertillon substitue des procédures fiables, rapides et régulières qui, à l'instar du relevé des longueurs osseuses, " ne sont nullement douloureuses et n'occasionnent pas une plus grande gêne que les mesures que nous prennent les tailleurs ${ }^{35}$ ». Véritables " juges d'instruction physique et mécanique ${ }^{36} "$, ces dernières ne sont pas employées pour extorquer de quelconques aveux. Les policiers ne répondent plus désormais aux dénégations identitaires par des réactions impulsives et agressives mais plutôt par des attitudes tempérées et distantes qui visent à bannir tout affrontement dans des relations directes de corps à corps et à limiter les échanges verbaux avec les personnes mensurées et photographiées ${ }^{37}$. Chaque jour, ils font preuve d'une plus grande retenue et maitrise d'eux-mêmes : ils accomplissent leurs tâches de façon répétitive "sans haine et sans passion [...] "sans considération de personne" ; formellement, de manière égale "pour tout le monde" 38 ". Comme le remarque alors Yves Guyot : " Un détenu a laissé échapper le mot : ça remplace le passage à tabac [...]. Au lieu d'avoir une police nerveuse, brutale, théâtrale, dramatique, aimant la réclame, il s'agit d'avoir une police tranquille ; faisant son œuvre en silence, fonctionnant avec des frottements doux, sans bruit, mais avec la précision d'une machine bien conçue, bien montée et composée de matériaux de premier choix $^{39}$ ».

13 Ainsi, anthropométrie, portraits signalétiques et autres relevés des stigmates corporels constituent des instruments de pouvoir d'une efficacité redoutable car même si la police n'inscrit plus sa présence à même la chair des individus (essorillements, tatouages, inscriptions au fer rouge, etc.), elle enregistre et conserve dorénavant avec minutie des "marques indélébiles et sans torture $\mathrm{A}^{40}$ ». Traces et signes qui, parce qu'ils révèlent le caractère absolument singulier de leur être, peuvent confondre les individus à tout moment. Le traitement symbolique de la question identitaire sur lequel repose le bertillonnage privilégie, au détriment de pressions purement physiques, une violence du savoir et du voir. Il engendre un archivage sans cesse plus perfectionné et centralisé de 
données qui, en facilitant l'ordonnancement d'une multitude d'individus sur le papier (fiches, photographies, etc.), confère à la police la capacité de dominer visuellement un " matériau humain " grâce auquel elle atteint chacun dans son intimité et procède à des opérations de totalisation très variées.

La constitution de cette " mémoire d'État ${ }^{41}$ » transforme en profondeur le rapport que délinquants et criminels entretiennent avec l'institution policière car à présent nul ne peut plus ignorer que cette dernière s'est de la sorte insensiblement immiscée au cœur même de son existence. Reposant sur le déploiement d'un appareillage technique qui rend possible, lors d'un bref moment de face à face, une mise en chiffres et en graphes de l'identité de chaque individu puis son classement sous la forme de fiches dans des boîtes, tiroirs et bibliothèque logiquement ordonnés, le bertillonnage dispense, par la suite, la police d'avoir à exercer une constante discipline ou surveillance sur son corps physique. Comme le souligne Claudine Dardy, " plus l'économie scripturaire devient complexe, plus elle se passe d'une présence charnelle ${ }^{42} »$. La maxime du criminaliste italien Cesare Beccaria selon laquelle "la loi doit suivre l'homme comme l'ombre suit son corps " ne relève plus alors vraiment de l'utopie puisque désormais les malfaiteurs ne peuvent qu'admettre la suprématie d'une raison d'État qui leur assène sur leur propre compte, en recourant à une forme de violence plus feutrée, une vérité à laquelle ils ne peuvent plus échapper. Le verrouillage policier de leur " double identitaire » abstraitement codifié doit les inciter à intérioriser l'idée qu'il ne leur est plus possible d'envisager de se soustraire aux sanctions qu'impliquent leurs actes délictueux. Objectif auquel veut parvenir un A. Bertillon qui reprend à son compte l'argumentaire développé, vingt ans plus tôt, par Louis Bonneville de Marsangy au sujet d'un casier judiciaire dont la gestion maladroite par le ministère de la Justice n'a, au début des années 1880, toujours pas permis de produire les effets escomptés par son inventeur : «Il est à présumer que les malfaiteurs, une fois mesurés et catalogués, y regarderont à deux fois avant de commettre ouvertement un crime quand ils auront compris qu'ils portent toujours sur eux, dans la longueur de leur tête, de leur pied et de leur doigt, leur nom en caractères indélébiles, et que pendant des années la moindre affaire avec la police pour ivresse, rixe, etc. pourrait amener la reconnaissance de leur identitée ${ }^{43}$ ».

Institué à l'origine comme l'unique remède apte à apporter une réponse adéquate aux problèmes d'identification soulevés par la lutte contre les récidivistes, le bertillonnage va peu à peu toucher des individus caractérisés par des statuts sociaux très divers. Selon Carlo Ginzburg, il tend à constituer « la tête de pont d'un projet d'ensemble, plus ou moins conscient, visant à exercer un contrôle généralisé et subtil sur la sociétét ${ }^{44}$ ».

Dès les premiers moments de leur mise en œuvre, les opérations d'identification de la Préfecture de police sont destinées à toutes les personnes passant par le Dépôt ${ }^{45}$. Conscient des éventuels excès qu'une telle pratique peut engendrer, A. Bertillon la considère cependant comme nécessaire étant donné que la politique répressive promue par l'État représente une priorité absolue qui, selon lui, légitime ce dernier à s'arroger discrétionnairement ce droit ${ }^{46}$.

En s'évertuant à présenter d'une façon récurrente ses procédés comme peu douloureux, l'inventeur de l'anthropométrie va parvenir à les faire appliquer à des catégories d'individus de plus en plus vastes, stigmatisées comme autant de figures de l'anormalité censées contenir en leur sein les germes de l'illégalisme. Au nom de la défense de la société et de l'intérêt général, c'est tout le monde de l'errance qui en devient une cible privilégiée. Dans une circulaire rédigée en juin 1887 à la demande du Parquet de Paris, A. 
Bertillon écrit : « Tout voleur, ou simple vagabond non nanti de papiers d'identité qui se déclarerait sans condamnations antérieures et qui n'indiquerait pas de référence d'identité sérieuse et d'un contrôle facile peut désormais être immédiatement l'objet d'une tentative de vérification de ce genre, accompagnant ou même précédant la demande de casier judiciaire ${ }^{47}$ ». Les malades mentaux n'échappent pas non plus à la suspicion des pouvoirs publics ${ }^{48}$. Tout comme le monde ouvrier, le plus souvent considéré par la police comme une " tourbe laborieuse " instable dont elle s'attache à enrayer les virulentes agitations afin qu'elles n'infectent pas moralement l'ensemble de la société. En 1883, A. Bertillon souligne que les usurpations d'identité sont surtout le fait d'individus que l'on trouve dans des " établissements insalubres, fabriques d'engrais, de blanc de céruse, etc..$^{49}$ ". Quatre ans plus tard, il présente, lors de l'Exposition Universelle qui se déroule dans la capitale, une collection photographique des principales marques corporelles à partir desquelles la police est capable d'identifier l'activité exercée par chaque ouvrier travaillant dans les différentes industries parisiennes !

Toutefois, au-delà de l'identification des individus que la police tient pour socialement suspects, le bertillonnage constitue un marqueur quasi infaillible de l'identité pouvant s'appliquer à tous ${ }^{50}$. S'inspirant des mensurations effectuées par la Préfecture de police, un journaliste parisien propose même, dès 1883 , un original système d'immatriculation qui permettrait à l'administration d'enregistrer la nation tout entière et de la " numéroter comme un troupeau de mouton ${ }^{51} »$. Si en France, pour des raisons sur lesquelles nous ne pouvons nous attarder longuement ici (émergence des empreintes digitales comme identifiant "concurrent ", enjeu de la protection des libertés individuelles, etc.), la police n'a jamais pu «embertillonner » toute la population, la pensée et l'œuvre de A. Bertillon lui ont néanmoins été d'une incontestable utilité dans tous ses projets de contrôle généralisé.

La définition rigoureuse d'un ensemble déterminé de propriétés physiques aptes à révéler la singularité de chacun, ainsi que la précision et la régularité avec lesquelles celles-ci sont dépeintes et relevées par les services d'identification de la Préfecture de police, concourent à réformer entièrement la manière dont, jusqu'alors, tous les fonctionnaires établissaient les signalements. Dans ce domaine, aux errements jadis caractérisés par le flou des éléments distinctifs du visage pris en compte et par l'approximation flagrante avec laquelle ceux-ci étaient libellés sur les documents de papier, A. Bertillon substitue une approche résolument descriptive et analytique qu'il préconise d'employer pour " toute pièce où la personnalité est établie dans l'intérêt de l'individu, des tiers, de l'État, tels que : passeports, livrets militaires [... $]^{52} »$. De plus, la stricte codification par A. Bertillon des règles relatives aux conditions d'élaboration des portraits photographiques prépare directement l'utilisation systématique de ceux-ci comme éléments essentiels d'identification sur tous les documents d'identité ${ }^{53}$ ainsi que leur progressive uniformisation sur ces derniers. Enfin, avec les travaux d'A. Bertillon sont surtout mis en œuvre des règles méthodiques de classement et d'archivage qui faisaient défaut à la police. Elles préfigurent une rationalisation de sa gestion des identités de papier qui reposera sur l'établissement d'un lien indéfectible entre des cartes et des fichiers ${ }^{54}$.

Le bertillonnage jette donc les bases d'une réorganisation « scientifique " de tout l'encartement policier. Dans un monde caractérisé par une complexification croissante des rapports sociaux où le critère de la nationalité, en tant que lien d'allégeance exclusif entre certains individus et un État les instituant en " homo nationalis ${ }^{55}$ ", tend à revêtir une importance primordiale, la police saura s'en inspirer pour mettre en carte des 
populations « à risque » dont la présence sur le territoire est de plus en plus perçue comme une menace pour la cohésion de la communauté nationale ${ }^{56}$. Si en 1883 , l'initiative du député Frédéric Thomas consistant à recommander pour tous les repris de justice condamnés à plus d'un an d'emprisonnement le port d'une carte d'identité distinctive est rejetée par les parlementaires ${ }^{57}$, quelques années plus tard, celles proposant d'encarter les étrangers et les nomades aboutiront. Ces derniers se verront par exemple attribuer, à partir de 1912, un carnet anthropométrique, véritable condensé des principales découvertes d'A. Bertillon, grâce auquel la police les suivra à la trace dans tous leurs déplacements en les « astreignant à justifier de l'emploi de leur temps pour ainsi dire jour par jour ${ }^{58}$ ». Dans le même temps, la police fera aussi appel aux apports du bertillonnage pour doter les Français d'une carte d'identité qui, tout en leur permet tant de décliner leur identité, « affirme à la fois l'existence d'une identité nationale partagée par ceux qui la possèdent et établit une séparation entre les citoyens et les autres ${ }^{59}$ ". La carte d'identité de Français instaurée par le préfet Leullier dans le département de la Seine à partir de 1921 est l'une des premières étapes décisives d'une telle entreprise.

21 Si certains ne voient dans cet encartement policier qu'un " sérieux progrès ${ }^{60}$ ", celui-ci traduit surtout une transformation manifeste du lien unissant l'individu à l'État-nation. Il ne lui est plus forcément imposé sur le mode de l'injonction car " c'est l'individu qui désormais sollicite les contraintes que le pouvoir lui fait subir. Pour faire partie des multiples catégories d'ayants droit construites par la société démocratique nationale, l'homme moderne doit constamment rendre compte de la légitimité de ses appartenances ${ }^{61}$ ». En conférant à la police la maîtrise de connaissances logiquement déterminées qui lui ont servi à parfaire ses modalités de mise en carte des individus, le bertillonnage a créé des conditions favorables à l'avènement et à la diffusion d'instruments sans cesse plus performants et standardisés l'autorisant à recourir « concrètement et quotidiennement [...] beaucoup moins souvent à la force physique qu'à la force symbolique ${ }^{62} »$. Instruments relevant de cette catégorie d'actes officiels de nomination dont Pierre Bourdieu remarque qu'ils constituent une des expressions les plus flagrantes de la violence symbolique monopolisée par l'État ${ }^{63}$. Ces morceaux de papier permettent en effet l'exercice d'une forme de contrôle social qui « impose [...] des comportements, perturbe une trajectoire sociale ${ }^{64} »$. Ils apparaissent comme autant de manifestations concrètes de la contrainte étatique, qui, désormais souvent intériorisées sur le mode de l'évidence parce qu'elles nous procurent aussi, par l'effet d'une véritable "illusion narcissique ${ }^{65}$ ", le sentiment d'exister et d'être reconnus là où nous sommes triés, contribuent amplement à la consolidation et à la pérennité d'un ordre social stable et pacifié en faisant advenir un consensus sur la façon d'apprécier l'identité de chacun.

Souvent négligés, ces documents représentent donc des modes de pénétration étatique du social qui ne sont nullement mineurs ou négligeables. Ils nous renseignent en outre également sur la capacité de l'État à monopoliser la production de l'identité nationale : en les utilisant les individus ne manipulent-ils pas « quotidiennement un savoir hétéroclite au travers duquel ils enregistrent le découpage étatico-national de l'espace et du temps comme une donnée fondamentale de leur biographie ${ }^{66} »$ ? Étudier les conditions de leur mise en forme (si elle permet d'en saisir certaines logiques, l'analyse du bertillonnage n'est bien sûr pas à elle seule suffisante pour les comprendre exhaustivement) ainsi que l'attachement que leur portent leurs possesseurs se révèle donc particulièrement nécessaire notamment pour tous ceux qui entendent apporter un début de réponse à cette interrogation essentielle ainsi formulée par Norbert Elias : "Pourquoi les liens 
affectifs qui nous attachent aux sociétés étatiques, aujourd'hui aux États nationaux, ontils la primauté sur ceux qui nous rattachent à d'autres configurations, alors qu'à d'autres stades de l'évolution les sociétés privilégiaient ceux qui nous reliaient aux villes, aux tribus, aux villages ${ }^{67}$ ?».

\section{BIBLIOGRAPHIE}

BERLIÈRE Jean-Marc, Le Préfet Lépine. Vers la naissance de la police moderne, Paris, Denoël, 1993.

BERTILLON Suzanne, Vie d'Alphonse Bertillon, inventeur de l'anthropométrie, Paris, Gallimard, 1984.

BRAUD Philippe, «La violence politique : repères et problèmes » dans La Violence politique dans les démocraties européennes occidentales, sous la direction de Philippe Braud, Paris, L'Harmattan, 1993.

DÉloye Yves, Sociologie historique du politique, Paris, La Découverte, 1997.

FouCAULT Michel, Résumé des cours. 1970-1982, Paris, Julliard, 1989.

GiNZBURG Carlo, « Signes, traces, pistes. Racines d'un paradigme de l'indice », Le Débat, novembre 1980.

NoIRIEL Gérard, La Tyrannie du national, Paris, Calmann-Lévy, 1991.

PHÉLINE Christian, « L’image accusatrice », Les Cahiers de la photographie, nº 17, 1985.

\section{NOTES}

*. Cette étude s'inscrit dans le cadre d'une thèse de doctorat de science politique actuellement en préparation à l'université Paris I-Panthéon-Sorbonne sous la direction de Philippe Braud.

1.Sur la trajectoire et l'entourage familial d'A. Bertillon, cf. Pierre Darmon, « Bertillon. Le fondateur de la police scientifique », L'Histoire, n 105 , novembre 1987.

2.En effet, même si une telle mesure a déjà été prévue pour les condamnés aux travaux forcés par une loi du 30 mai 1854, de nombreuses voix s'élèvent dans les années 1870-1880 pour qu'elle soit étendue à une plus large frange de la population criminelle et délinquante. Par exemple une pétition organisée par la loge franc-maçonnique parisienne « Le Travail et la Persévérante amitié » demandant à ce que soient exilés les délinquants ayant subi plusieurs condamnations obtient en 1880 plusieurs milliers de signatures, cf. Robert Badinter, La Prison Républicaine, Paris, Fayard, 1993, p. 112.

3.Cf. à ce sujet Anne-Claude Ambroise-Rendu, « Un certain écho du monde. Propositions pour une lecture des faits divers de presse », Recherches contemporaines, $n^{\circ} 3$, 1995-1996, p. 24.

4.Bernard Schnapper, «La récidive, une obsession créatrice au XIX $x^{e}$ siècle », dans Voies nouvelles en histoire du droit : la justice, la famille, la répression pénale, $\mathrm{XVI}-\mathrm{XX}{ }^{e}$ siècle, Paris, P.U.F., 1991. 
5.Comme le soulignait déjà $\mathrm{A}$. Bertillon en 1881 : «Quelle que soit la peine réservée aux récidivistes, on doit désirer, pour qu'elle remplisse son plein effet préventif, qu'elle ne puisse être éludée et que l'individu déjà condamné soit sûr, s'il reparaît devant la justice, qu'on reconnaîtra son identité ", A. Bertillon, Une application pratique de l'anthropométrie sur un procédé d'identification permettant de retrouver le nom d'un récidiviste au moyen de son seul signalement et pouvant servir de cadre pour une classification de photographies à la Préfecture de police de Paris, à la Sûreté générale, au ministère de la Justice..., Paris, G. Masson, 1881.

6.Expression qu'il emploie dans une lettre adressée au docteur Variot au sujet du procédé mis au point par ce dernier en vue de « détatouer » les criminels. Procédé qui, selon A. Bertillon, « à défaut du cœur et de la cervelle blanchit incontestablement la peau », Archives de la Préfecture de Police de Paris (ci-après APP) 191.

7.M. Foucault, Résumé des cours. 1970-1982, Paris, Julliard, 1989, p. 99.

8.Id., « La volonté de savoir », ibid., p. 10.

9.Cf. G. Noiriel, La Tyrannie du national, Paris, Calmann-Lévy, 1991, p. 313.

10.M. Foucault, Surveiller et punir, Paris, Gallimard, 1975, p. 139.

11.Expression utilisée par A. Bertillon dans un de ses rapports datant de 1896, APP DB 47.

12.Véritable antichambre des prisons parisiennes, le Dépôt est le lieu où sont provisoirement placées en détention toutes les personnes arrêtées par la police avant d'être déférées devant la Justice.

13.Hugues Le Roux, Le Chemin du crime, Paris, Harvard, 1889, p. 265.

14.A.Bertillon, L'Anthropométrie judiciaire à Paris en 1889. Installation et plan des locaux, récents perfectionnements. Une expérience de sociologie bureaucratique, Paris, G. Steinheil, 1890, p. 13.

15.M. Foucault, « L'œil du pouvoir », reproduit dans Jeremy Bentham, Le Panoptique, Paris, Belfond, rééd. 1977, p. 10.

16.Article « Au Dépôt. Comment on reconnaît les récidivistes » du journal La Lanterne, 23 avril 1883.

17.Voir par exemple l'ouvrage Les Populations dangereuses et la misère sociale (Paris, E. Dentu, 1872) dans lequel P. Céré entend démontrer l'essor irrésistible des pratiques délictueuses et criminelles en s'évertuant à comptabiliser l'ensemble des individus dangereux sur le territoire national. Voir encore celui de J. Reinach intitulé Les Récidivistes (Paris, G. Charpentier, 1882) dans lequel l'auteur écrit page 12 : « Ils sont une armée compacte, une association contre la société, une ligue contre la loi, un corps de réfractaires dont la profession est d'attenter à la sûreté et à la propriété publique ». Les titres d'autres livres publiés à cette période sont particulièrement significatifs : La France criminelle de Henri Joly (Paris, Le Cerf, 1889), L’Armée du crime de Félix Platel (Paris, V. Harvard, 1890), etc.

18.Gustave Macé, Le Service de la Sûreté par son ancien chef, Paris, G. Charpentier, 1891, p. 378.

19.0bjectif principal d'un pouvoir disciplinaire dont Hubert Dreyfus et Paul Rabinow rappellent qu'il cherche surtout à " produire un être humain qu'on puisse traiter comme un corps "docile" ", Michel Foucault, un parcours philosophique, Paris, Gallimard, 1984.

20.Suzanne Bertillon, Vie d'Alphonse Bertillon, inventeur de l'anthropométrie, Paris, Gallimard, 1941, p. 136.

21.Expression employée par A. Bertillon dans un de ses rapports en date du 6 juin 1894, APP BA 960. 
22.A. Bertillon en est lui-même conscient puisqu'il écrit : « Nulle part l'acte de reconnaissance ne se fait d'une façon aussi brutale que dans l'exercice de la photographie judiciaire ", La Photographie judiciaire, Paris, Gauthier-Villars, 1890, p. 3.

23.Ibid., p. 60.

24.Christian Phéline, "L'image accusatrice », Les Cahiers de la photographie, n 17, 1985, p. 105.

25.M. Foucault, Surveiller et punir..., op. cit., p. 55.

26. La France, Le Journal des débats, Le XIX ${ }^{e}$ siècle, Le National, La Patrie, etc. se félicitent de l'efficacité de ses travaux.

27.A. Bertillon, «L'identité des récidivistes et la loi de la relégation », Revue politique et littéraire, $\mathrm{n}^{\circ} 17,28$ avril 1883, p. 22.

28.Le seul cas de résistance collective dont nous avons pu retrouver la trace est celui relaté par Edmond Locard : «J'ai entendu raconter, vers 1903, par un aide de Alphonse Bertillon, que les individus montant de l'étage où se fait l'anthropométrie à celui où l'on opère la photographie signalétique s'entendirent pour donner chacun au policier chargé de ce dernier service le nom de son voisin, ainsi, la photographie du second fut collée sur la fiche du premier, celle du troisième sur la fiche du second et ainsi de suite ", Traité de criminalistique, Lyon, Desvignes, 1931, volume III, p. 593.

29.A.Bertillon, Identification anthropométrique, instructions signalétiques, Melun, Imprimerie administrative, 1893 , p. 7.

30.M. Foucault, « Il faut défendre la société », Cours au collège de France, Paris, Seuil, 1997, p. 26.

31.Pour une approche générale de ce phénomène, cf. notamment Jean-Marc Berlière, «La professionnalisation : revendication des policiers et objectif des pouvoirs au début de la Troisième République ", Revue d'histoire moderne et contemporaine, $\mathrm{n}^{\circ} 37,1990, \mathrm{p} .398-428$. 32.Pour une description de certaines d'entre elles, comme le « moutonnage ", cf. Martine Kaluszynski, « Alphonse Bertillon et l'anthropométrie », dans Maintien de l'ordre et police en France et en Europe au XIX $X^{e}$ siècle, ouvrage collectif, Paris, Créaphis, 1987, p. 271.

33.Cf. J.-M Berlière, Le Préfet Lépine. Vers la naissance de la police moderne, Paris, Denoël, 1993, p. 156.

34.Dans certains cas, les victimes se transforment même en complices. À cet égard, l'exemple de la prime de cinq francs allouée, depuis 1844, par le préfet de police à tout policier ou gardien de prison démasquant un repris de justice sous un faux nom est particulièrement révélateur d'une telle collusion : les intéressés se mettent quelquefois d'accord pour la partager et A. Bertillon de remarquer que l'argent ainsi distribué aux fonctionnaires « est dépensé chez le marchand de vin, en tournées réciproques, le jour même où il leur est versé » (note d'A. Bertillon à M. Taylor, commissaire de police, chef du service de Sûreté, 30 juillet 1887, APP 191).

35.A.Bertillon, Une application pratique de l'anthropométrie..., op. cit., p. 20.

36.Formule de Gabriel Tarde, La Philosophie pénale, Paris, G. Masson, 1891, p. 451.

37. «Lorsque la conviction de l'agent est assise, il transmet le renseignement à l'autorité compétente, la plupart du temps, sans même daigner informer le sujet du résultat de ses recherches et de ses constatations », A. Bertillon, Sur le fonctionnement du service des signalements anthropométriques, Lyon, A. Storck, 1888, p. 15.

38. Max Weber, Économie et Société, volume I, Paris, Plon, rééd. 1995, p. 300.

39.Y. Guyot, La Police, Paris, G. Charpentier, 1884, p. 433.

40.Termes utilisés par A. Bertillon dans une correspondance adressée au préfet de police le $1^{\mathrm{er}}$ décembre 1879, APP 191. 
41.G. Noiriel, La Tyrannie..., op. cit., p. 164.

42.C. Dardy, « Racontez-moi des histoires de papier », Actions et Recherches sociales, $\mathrm{n}^{\circ} 2$, juin 1990, p. 9.

43.A. Bertillon, « L'identité des récidivistes... », loc. cit., p. 20. Dans son ouvrage intitulé De l'amélioration de la loi criminelle en vue d'une justice plus prompte, plus efficace, plus généreuse et plus moralisante, L. Bonneville de Marsangy écrivait quant à lui à propos du casier judiciaire : «Si voulant usurper les droits et les attributions dont leur délit les a rendu indignes, ils osaient se présenter au forum ; s'ils osaient approcher de l'urne électorale ou monter sur le siège du juge, s'ils osaient se mêler furtivement dans les rangs de nos braves soldats, de nos généreux citoyens, défenseurs de l'ordre et des lois ; si surtout ils osaient venir dans nos clubs prêcher la révolte et l'anarchie, je veux qu'alors l'autorité, fixant sur eux son œil sévère, leur fasse comprendre qu'elle connaît leur secret, qu'elle a sous la main leur exacte biographie, et qu'ainsi avertis, ils rentrent aussitôt dans le silence et l'obscurité dont ils n'auraient jamais dû sortir ", Paris, Librairie du Conseil d'État, 1864, volume 1, p. 667.

44.C. Ginzburg, « Signes, traces, pistes. Racines d'un paradigme de l'indice », Le Débat, novembre 1980, p. 37.

45.Cette pratique suscite d'ailleurs la réprobation du Conseil municipal de Paris qui souligne les abus auxquels elle peut conduire puisque de nombreux individus, seulement détenus préventivement, donc parfois coupables d'aucun délit avéré, sont ainsi photographiés par la police et voient leur portrait « figurer dans une galerie sinistre à côté des malfaiteurs de tout genre ", Rapport Leveau concernant le projet de budget spécial de la Préfecture de police de Paris, 1880.

46. «Quelques personnes pourront objecter : mais à quoi bon mesurer, en règle générale, tous les individus conduits au Dépôt ? Pourquoi ne pas se borner à mensurer ceux qui auront été retenus par le magistrat du Petit Parquet ? La réponse est simple : c'est que dans le flot des individus qui passent au Dépôt quelques heures seulement (et que le Petit Parquet peut ne pas retenir en raison du peu d'importance du délit commis) se trouvent un grand nombre de condamnés par défaut ou des contumaces recherchés par les Parquets de province, ou encore des expulsés, des déserteurs ou des gens qui dissimulent leur identité en raison de leurs antécédents judiciaires », rapport d'A. Bertillon datant de 1895, APP DB 47.

47.APP 191.

48.Dans un rapport (non daté) qu'il fait parvenir à $M$. Naudin, chef de la première division de la Préfecture de police, A. Bertillon se prononce en faveur de la mensuration de tous les aliénés afin de repérer d'éventuels « simulateurs », cf. APP 191.

49.A. Bertillon, « L'identité des récidivistes... », loc. cit., p. 2.

50.En 1883, A. Bertillon obtient l'autorisation d'y recourir à la morgue. Par la suite, il sera aussi parfois utilisé par la Préfecture de police pour constater la «non-identité d'honorables citoyens [confondus] avec les véritables auteurs de crimes et délits qui ont usurpé un état civil », Conseil municipal de Paris, rapport présenté par M. Achille sur le projet de budget spécial de la Préfecture de police (1918).

51. «L'anthropométrie à la morgue », Feuilleton de la ville de Paris, 11 octobre 1883.

52.Définition du terme « signalement » par A. Bertillon dans le Dictionnaire des sciences anthropométriques, APP 191.

53.À propos des passeports, A. Bertillon écrit : « Pourquoi ne pas compléter les signalements par l'adjonction d'une photographie collée sur le passeport même et réunie 
à celui-ci par un timbre sec ? ", « La Préfecture à l'Exposition d'Amsterdam », La Nature, 25 août 1883, p. 106.

54.À cet égard, l'introduction de la numérotation par A. Bertillon pour améliorer les performances de ses classifications mérite d'être signalée : « Pour éviter toute confusion dans la transcription des états civils, et pour faciliter le classement ultérieur des clichés, on attribuera à chaque sujet photographié un numéro d'ordre, suivant son rang d'inscription sur le registre spécialement destiné au contrôle des opérations photographiques [...] Cette indication reproduite ainsi par la photographie elle-même permettra, en se rapportant au registre, de retrouver facilement le nom, l'état civil et les mensurations de chaque sujet ", A. Bertillon, La Photographie judiciaire..., p. 79.

55.Expression employée par Étienne Balibar dans « La forme de la nation : histoire et idéologie » dans Étienne Balibar et Immanuel Wallerstein, Race, nation, classe. Les identités ambiguës, Paris, La Découverte, 1997, p. 126.

56.Comme le remarque justement J.-M.Berlière, la Troisième République « substitue à la devise républicaine celle de "liberté, égalité, carte d'identité" ", « La généalogie d'une double tradition policière » dans La France de l'affaire Dreyfus sous la direction de Pierre Birnbaum, Paris, Gallimard, 1994, p. 217.

57.Cf. à ce propos l'article de F. Thomas "Question des récidivistes. La carte d'identité ", Revue politique et littéraire, $\mathrm{n}^{\circ}$ 21, 24 novembre 1883.

58.Propos tenus par M. Sébille, contrôleur des recherches au ministère de l'Intérieur, lors de la séance de la Société générale des prisons du 17 juin 1914, Revue pénitentiaire et de droit pénal, $\mathrm{n}^{\circ}$ 6-12, juin-décembre 1914, p. 817.

59.Yves Déloye, Sociologie historique du politique, Paris, La Découverte, 1997, p. 77.

60.Marcel Sicot, Fausses et vraies identités, Paris, Hachette, 1967, p. 126.

61.G. Noiriel, La Tyrannie..., op. cit., p. 313.

62.Dominique Montjardet, Ce que fait la police. Sociologie de la force publique, Paris, La Découverte, 1996, p. 20.

63.Cf. P. Bourdieu, Choses dites, Paris, les Éditions de Minuit, 1987, p. 161.

64.Philippe Braud, «La violence politique : repères et problèmes » dans La Violence politique dans les démocraties européennes occidentales, sous la direction de Ph. Braud, Paris, L'Harmattan, 1993, p. 15.

65.Cf. J. Borie, Mythologies de l'hérédité au XIX siècle, Paris, Galilée, 1981, p. 65-67.

66.Hubert Pérès, «Le village dans la nation française sous la Troisième République. Une configuration cumulative de l'identité » dans Cartes d'identité. Comment dit-on nous en politique, sous la direction de Denis-Constant Martin, Paris, P.F.N.S.P., 1994, p. 225. 67.N. Elias, Qu'est-ce que la sociologie ?, Paris, Éditions de l'Aube, 1991, p. 168-169.

\section{RÉSUMÉS}

Au début de la III ${ }^{e}$ République, le bertillonnage est instauré à la Préfecture de police de Paris afin de faciliter la reconnaissance étatique de l'identité des récidivistes. Technique disciplinaire d'assujettissement des corps, il est aussi à l'origine d'une réorganisation rationnelle de l'encartement policier autorisant un mode d'imposition de la domination d'ordre symbolique*. 
The building of identity

IDENTITY AND THE BERTILLON SYSTEM : BETWEEN CULTURAL AND SYMBOLIC VIOLENCE

At the beginning of the Third Republic, the Bertillon system was established at the headquarters of Paris in order to facilitate the state recognition of the repeat offenders' identity. Being a disciplinary technique consisting in the subjection of bodies, it is also the origins of a rational reorganisation of the police cardindexing which allows a way to impose a symbolic domination.

\section{AUTEUR}

\section{PIERRE PIAZZA}

Pierre PIAZZA est rattaché au département de Science politique de l'Université de Paris I (Panthéon-Sorbonne). Il prépare une thèse de doctorat consacrée à la carte nationale d'identité, sous la direction de Philippe Braud. Pedrovirgo(at)aol.com 